\title{
Towards sector-based attribution using intra-city variations in satellite-based emission ratios between $\mathrm{CO}_{2}$ and $\mathrm{CO}$
}

Wu et al.

Correspondence to DienWu (dienwu@caltech.edu) 


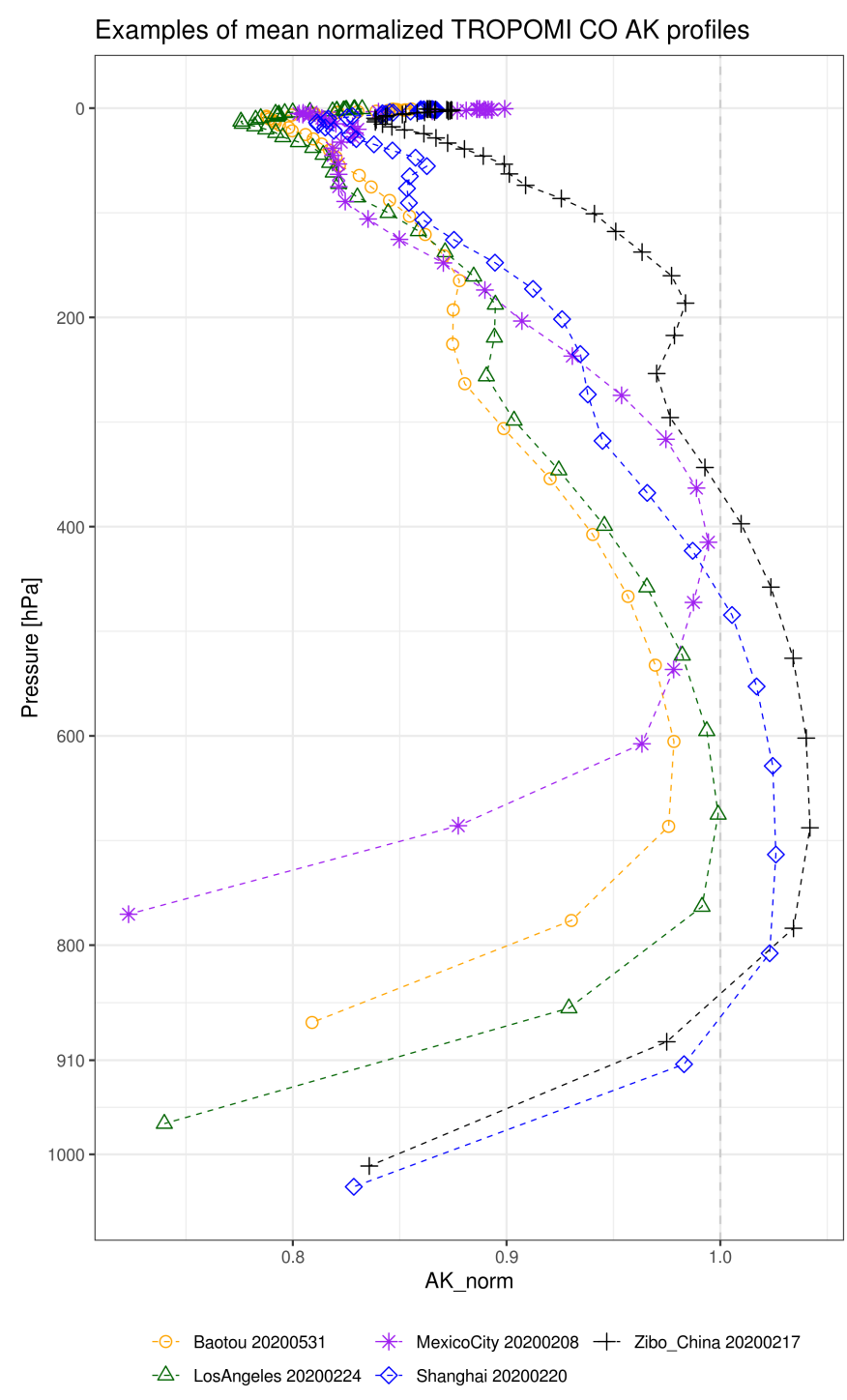

Figure S1. Examples of normalized averaging kernel [unitless] profiles from TROPOMI XCO, average over multiple soundings for one overpass per city. Colors denote the different overpasses. Normalized AKs are mostly lower than one for levels near the surface. 
a) Normalized AK profiles from sensors

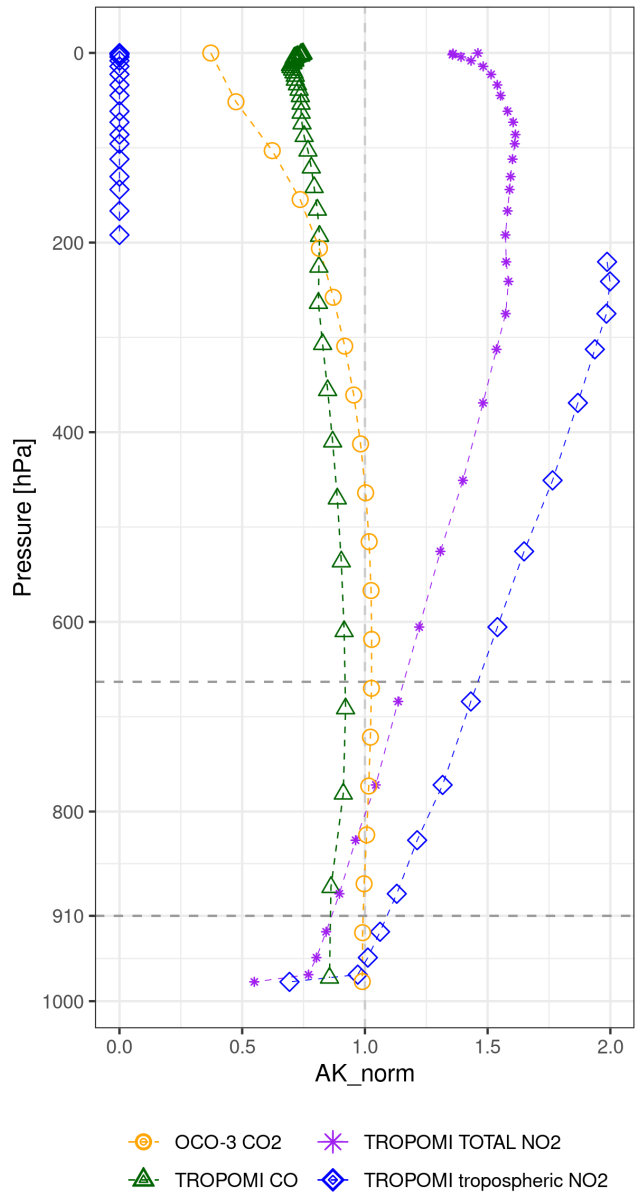

b) Weighted footprint using OCO-3 profiles

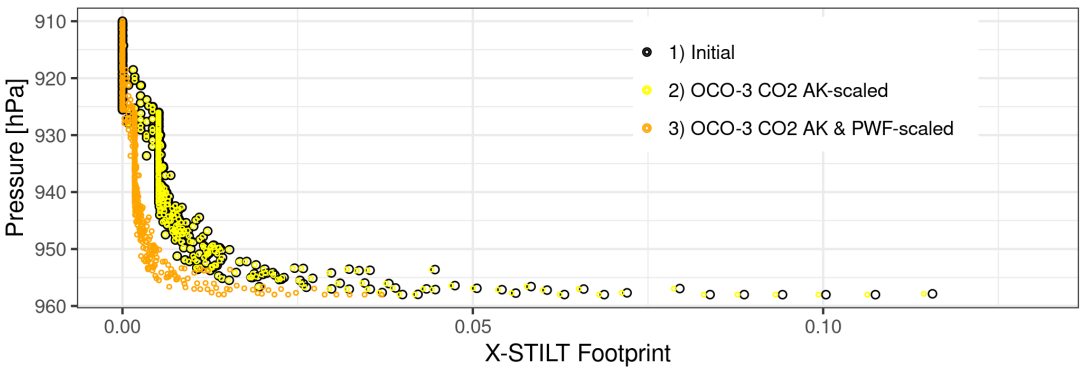

c) Weighted footprint using TROPOMI CO profiles

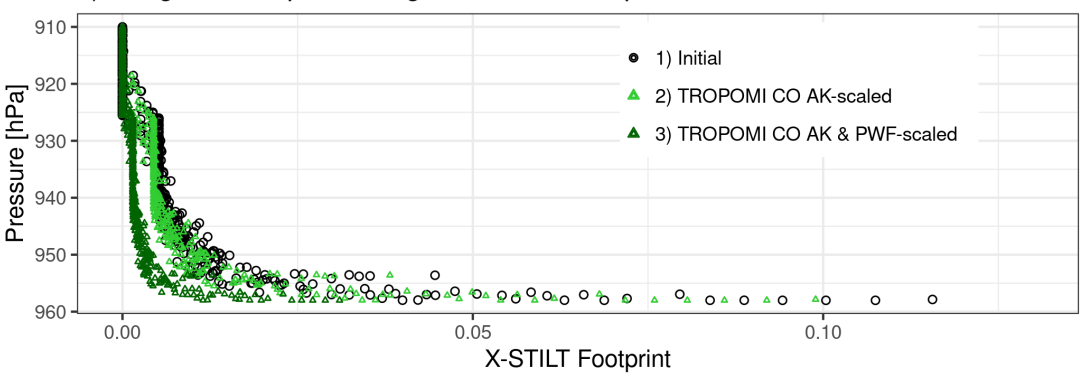

d) Weighted footprint using TROPOMI tNO2 profiles

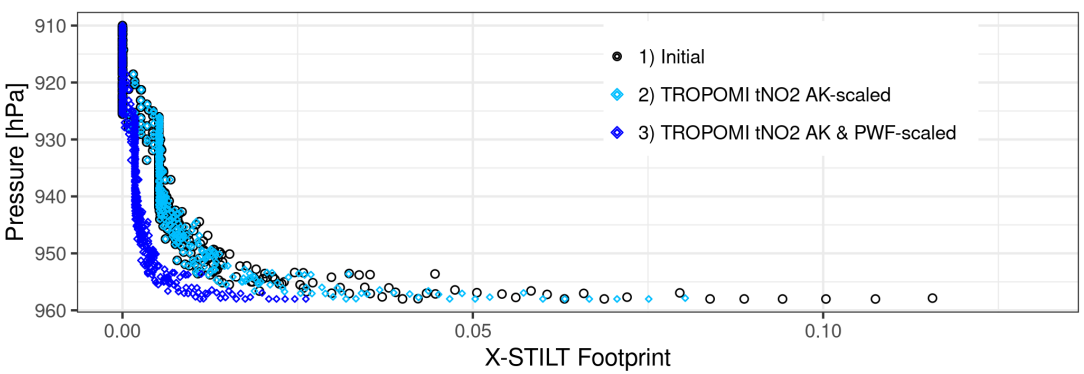

Figure S2. Demonstration of how AK and PWF are incorporated into the calculation of STILT footprint for every vertical level. a) Example of normalized AK profiles from OCO-3 $\mathrm{XCO}_{2}$, TROPOMI XCO and $\mathrm{NO}_{2}$. For TROPOMI $\mathrm{NO}_{2}$ AKs, profiles for both the total (purple stars) and tropospheric column (blue diamonds) are shown. b-d) The vertical weighting procedure of initial STILT footprint (black circles) using sensor-specific AKs (light yellow/green/blue circles) and using both AKs and pressure weighting functions (dark orange/green/blue circles). The footprint values are tied to every air parcels originating from the vertical column of the sounding. Footprints for air parcels at different altitudes will be weighted accordingly given the sensor-specific profiles. Note that this weighting procedure has now been implemented in X-STILT. Please refer to the data and code availability section in the main text for using the model. 
Sounding-specific column footprint [ppm / (umol m-2 s-1)] over LosAngeles on 20200224
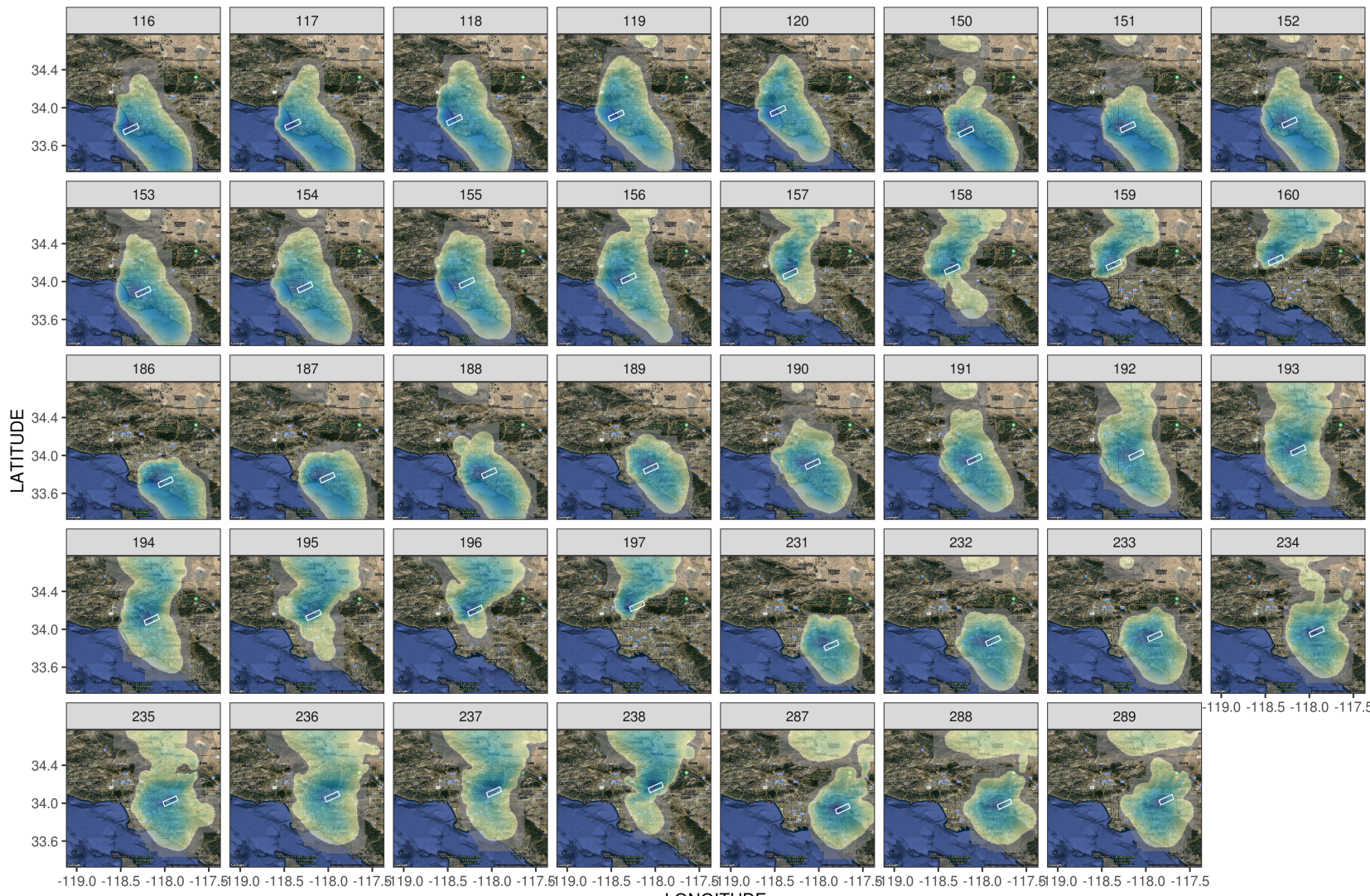

$19.0-118.5-118.0-117.5$ LONGITUDE

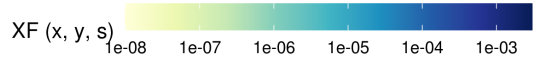

Figure S3. Spatial maps of column footprints $X F_{C O_{2}}(x, y, s)\left[\mathrm{ppm} /\left(\mu \mathrm{mol} \mathrm{m}{ }^{-2} \mathrm{~s}^{-1}\right)\right]$ from X-STILT over LA on Feb 20, 2020. These column footprints are unique to each satellite sounding given unique source-receptor relation. Note that the column footprint is initially generated for every OCO-2/3 soundings, but later aggregated to the TROPOMI scale (white rectangles). Column footprint for TROPOMI XCO can be slightly different from these maps due to discrepancies in AK profiles and atmospheric transport. The underlying hybrid maps were created using ggmap library in R that adopted the Google Maps (copyright: Map data @2021 Imagery @2021 TerraMetrics). 
Sounding-specific industrial contribution [\%] over LosAngeles on 20200224
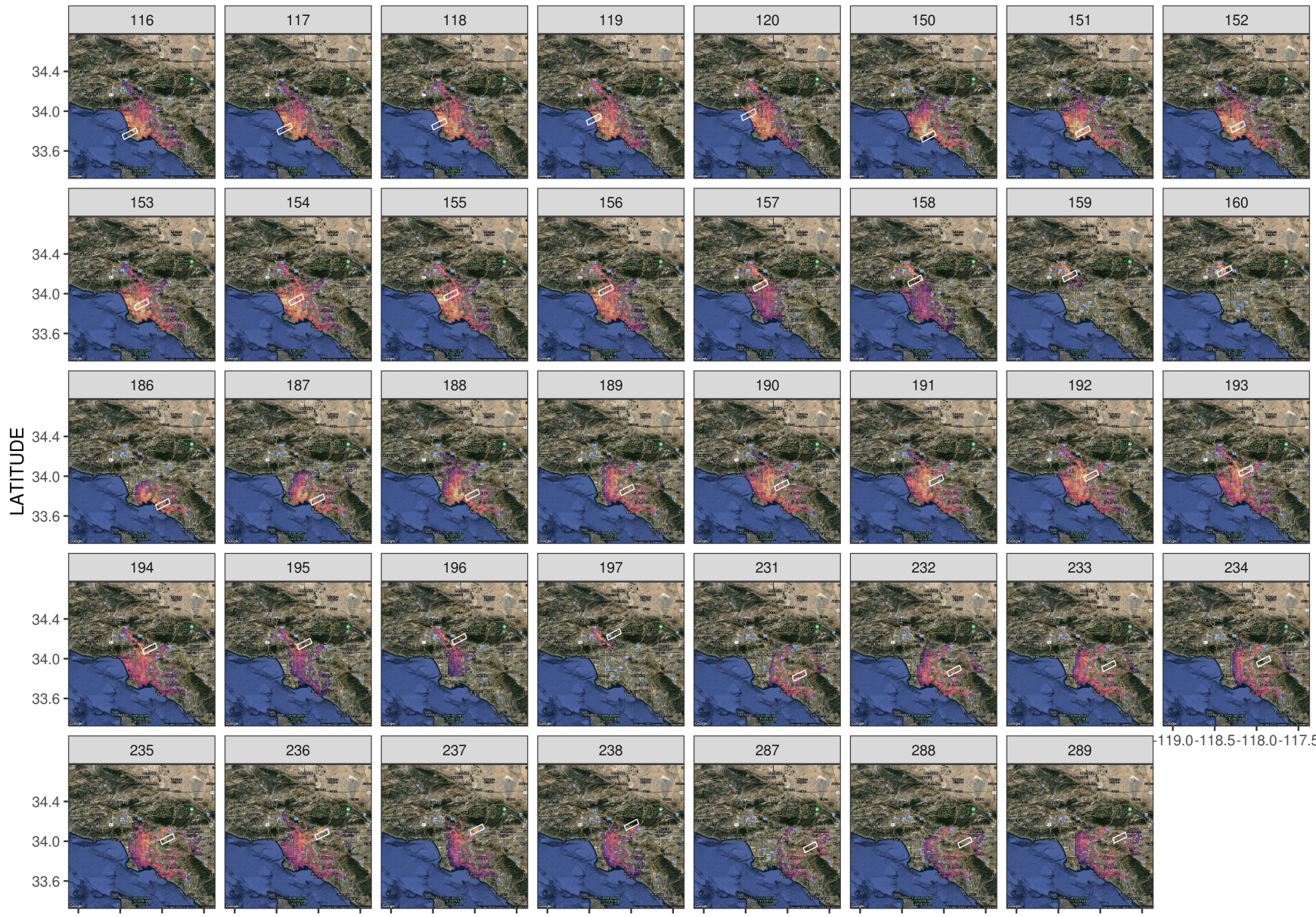

$119.0-118.5-118.0-117.5$

$-119.0-118.5-118.0-117.519 .0-118.5-118.0-117.519 .0-118.5-118.0-117.519 .0-118.5-118.0-117.519 .0-1$
LONGITUDE

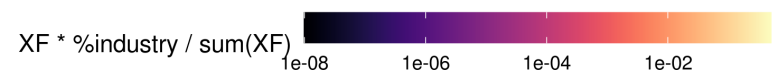

Figure S4. Spatial maps of the column footprint- normalized industry fraction $\left(P_{i n d}(x, y)\right.$ in $\left.\%\right)$ over LA on Feb 20, 2020. Since the initial industry fraction from WUDAPT is normalized by the column footprint, these resultant normalized fraction $P_{\text {ind }}(x, y)$ informs the influence on the observation at a given sounding (white rectangle) due to heavy industry. Lighter the color, stronger impact from heavy industry in LA. The underlying hybrid maps were created using ggmap library in R that adopted the Google Maps (copyright: Map data @2021 Imagery @2021 TerraMetrics). 

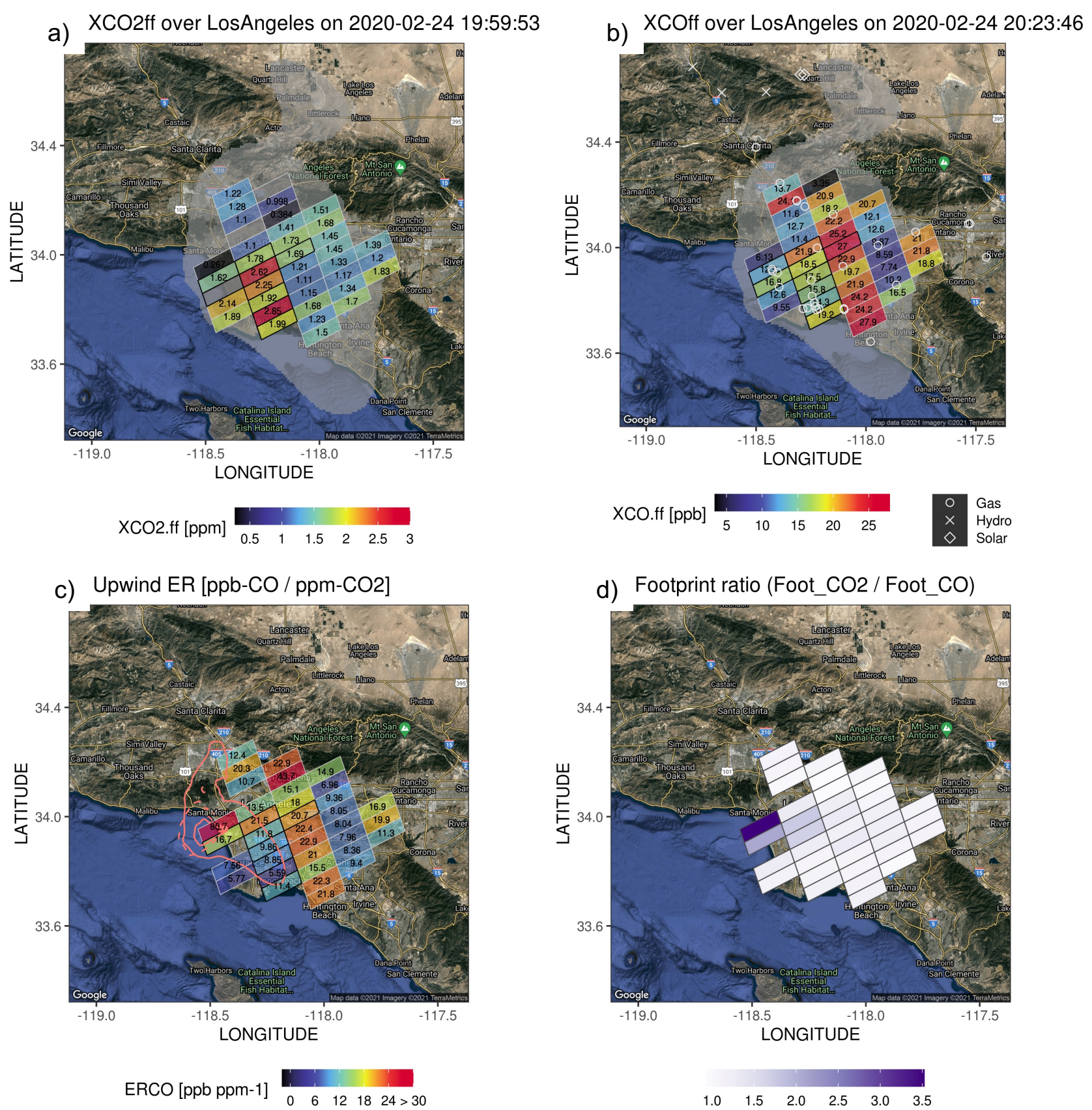

Figure S5. An example of the sounding-level information over LA on Feb 24, 2020. a, b) Same as Fig. 2bc, but with the inclusion of urban-background biogenic gradient. Since biogenic gradient for the city center is usually positive, the $\mathrm{XCO}_{2}$ enhancements shown in a) are slightly smaller than Fig. $2 \mathrm{~b}$. c) Spatially-explicit emission ratio following Eqs. 1 and 2 in the main text. The industry-dominant soundings are highlighted with black outlines. d) The ratio in the spatial sum of the column footprints between OCO-3 $\mathrm{XCO}_{2}$ and TROPOMI XCO (i.e., $\gamma$ foot in Eq. 2). Two sets of footprint totals are different because of the difference in AK and wind fields. Higher the footprint ratio, larger the discrepancies between the enhancement ratio and the emission ratio (Eq. 2). The underlying hybrid maps were created using ggmap library in R that adopted the Google Maps (copyright: Map data @2021 Imagery @2021 TerraMetrics). 

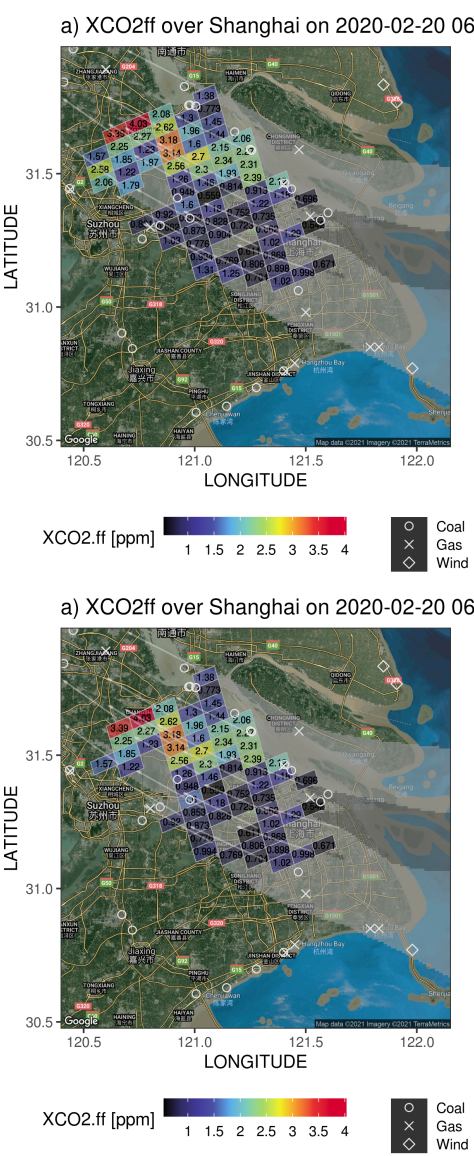
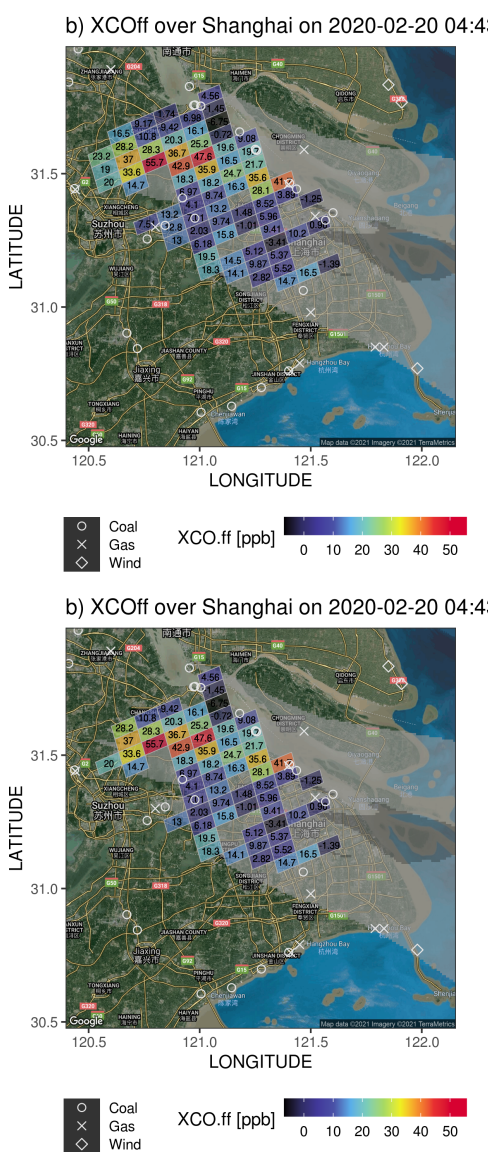

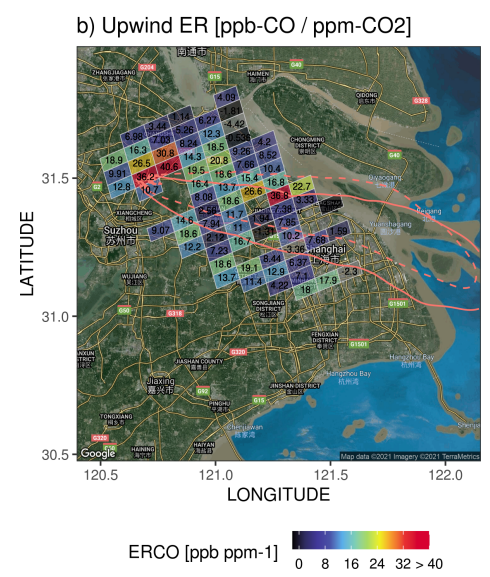

b) Upwind ER [ppb-CO / ppm-CO2]

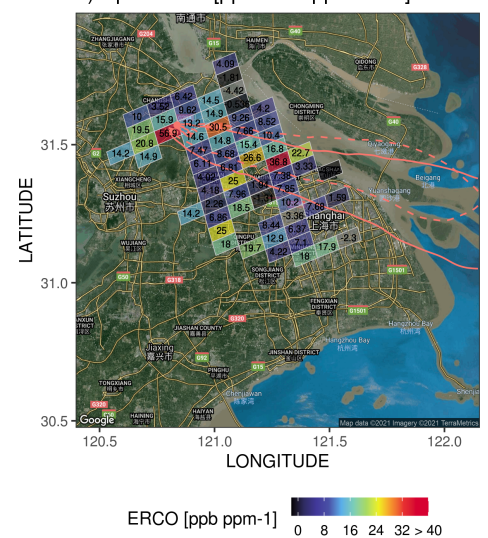

Figure S6. An example of $\mathrm{XCO}_{2}$ and XCO FF enhancements over Shanghai on Feb 20, 2020. The first and the second row presents the FF enhancements and sounding-level $\mathrm{ER}_{\mathrm{CO}}$ before and after the plume shift, respectively. The gray regions indicate the source regions modeled by X-STILT. During the manual shifting procedure, $\mathrm{XCO}_{2}$ enhancements move southward by 0 to 2 grids (depending on their longitudes) while XCO enhancements remain the same, leading to changes in the spatial distribution of $\mathrm{ER}_{\mathrm{CO}}$ after re-aligning the urban plumes during two overpass times. The correlation coefficient increases by $10 \%$ after the shift. The underlying hybrid maps were created using ggmap library in R that adopted the Google Maps (copyright: Map data @2021 Imagery @2021 TerraMetrics). 
Histogram of modeled biogenic XCO2 anomalies [ppm] per OCO sounding
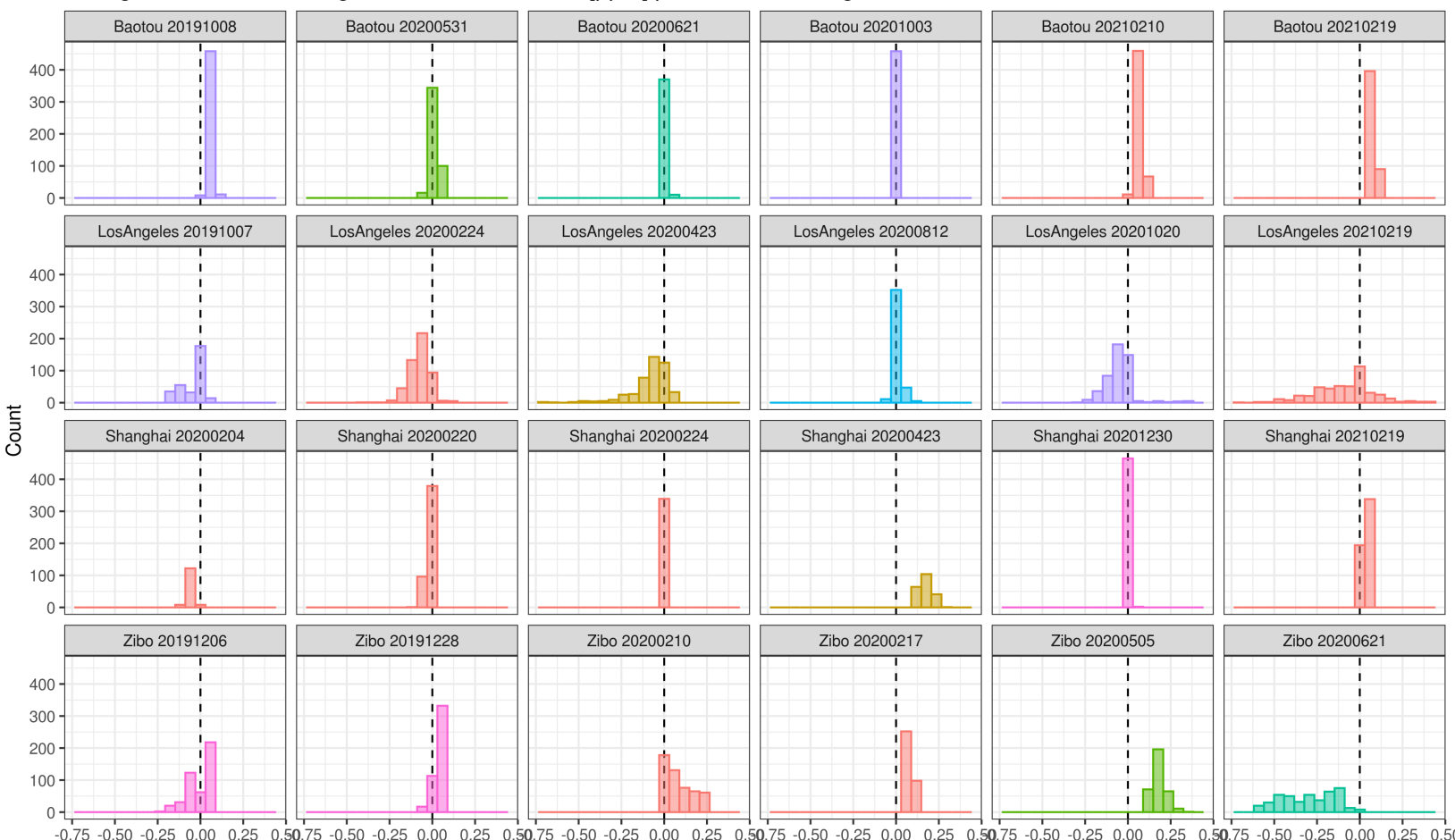

Modeled XCO2.bio [ppm]

Figure S7. Histogram of modeled biogenic $\mathrm{XCO}_{2}$ anomalies [ppm] at each OCO-2/3 sounding location with color code for different months. These biogenic anomalies are calculated as the spatial sum of the hourly column footprint and hourly NEE fluxes from a biospheric model. If the OCO-3 overpass time is close to the noon, daytime carbon sink dominant leading to negative anomalies at the overpass time (e.g., dark green polygons in Supplementary Fig. S8a,c). However, if the OCO-3 overpass time approaches morning or during the wintertime, the carbon uptake is weaker and the nighttime respiration dominant, leading to net positive anomalies. The biogenic signals for Baotou and Shanghai are smaller compared to LA and Zibo. Note that these biogenic anomalies are further averaged to the TROPOMI scale. We stress again that the urban-background contrast of these anomalies (e.g., brown colors in Supplementary Fig. S8b,d) modify the anthropogenic enhancements, not the anomalies themselves. We further display the modeled biogenic anomalies and their urbanbackground gradient on a map for the two cases with strongest biogenic gradients in Supplementary Fig. S8 to illustrate the difference. 

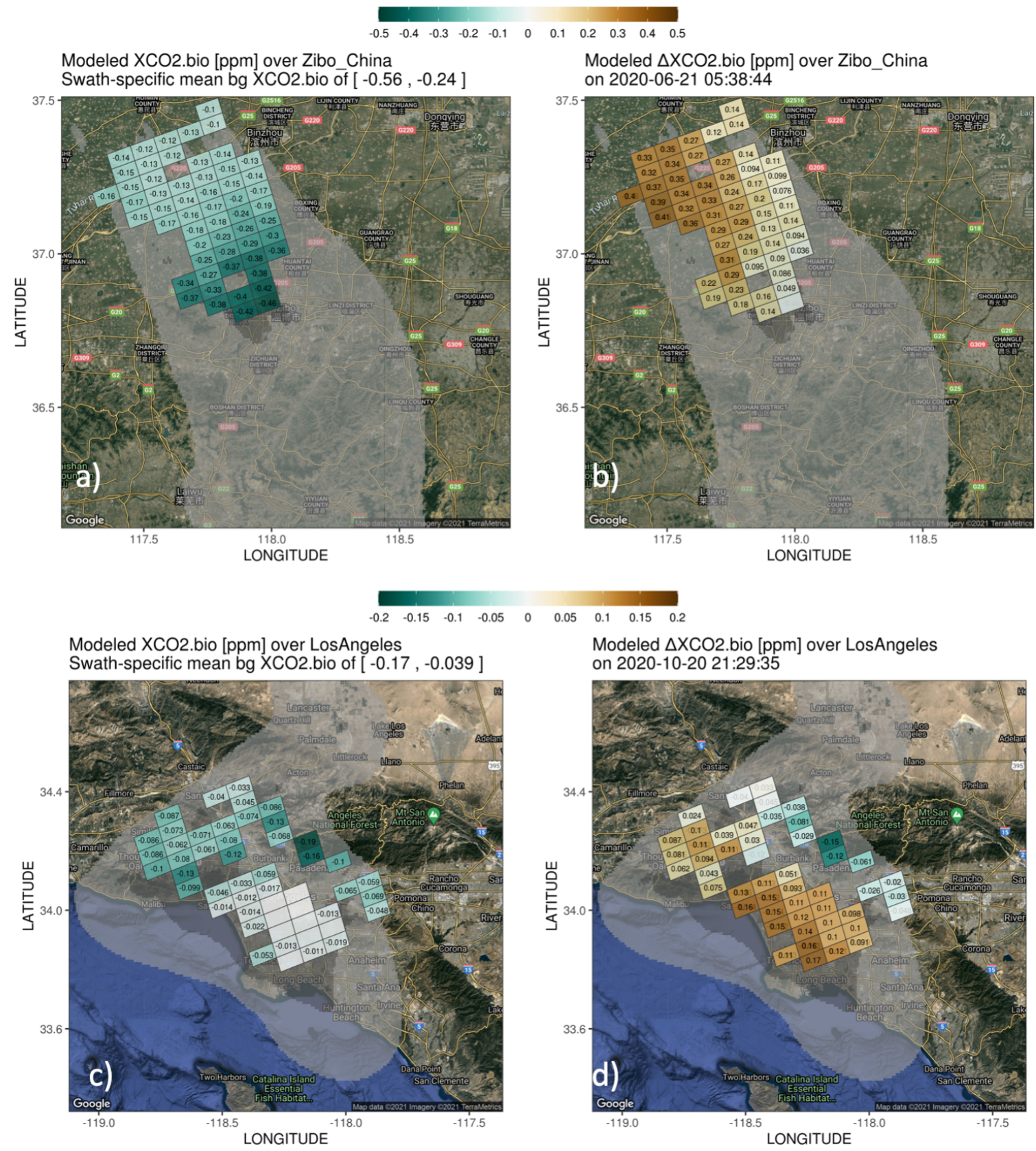

Figure S8. Spatial maps of modeled biogenic anomalies (a, c) and urban-background contrast (b, d) tied to each downwind satellite sounding for Zibo on June 21, 2020 and LA on Oct, 20, 2020. For example, we chose the northern land outside the LA basin as the background. The background biogenic contribution is more negative than those biogenic anomalies in the basin. As a result, the gradient $\left(\delta X_{b i o}\right)$ for soundings in the basin now becomes positive. The underlying hybrid maps were created using ggmap library in R that adopted the Google Maps (copyright: Map data @2021 Imagery @2021 TerraMetrics). 

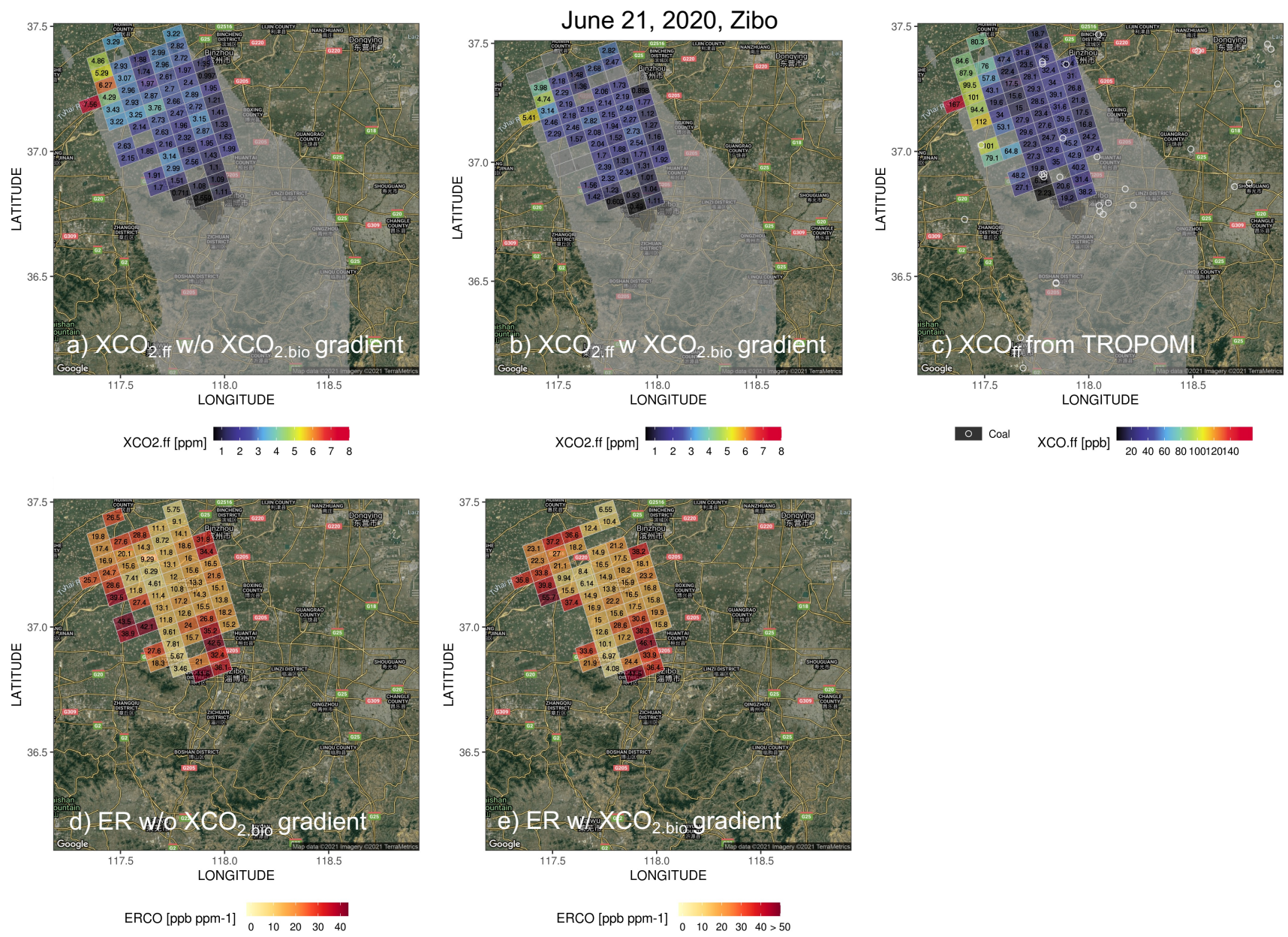

Figure S9. Spatial maps of derived FF enhancements (ppm- $\mathrm{CO}_{2}$ in a, b and ppb-CO in c) and $\mathrm{ER}_{\mathrm{CO}}\left(\mathrm{ppb}_{\mathrm{ppm}}{ }^{-1}\right.$ in d, e) with or without the account of the urban-rural biogenic gradient over Zibo on June 21, 2020. Only soundings within the urban plume are displayed. The light grey shading denote the near-field source region (defined by the X-STILT column footprint) with respect to those displayed soundings, given wind coming from the south. Considering the wind direction, observations further south of the displayed soundings are regarded as the background region (with latitude $<36.8^{\circ} \mathrm{N}$, not plotted). As shown in Fig. S8, the biogenic gradient for soundings within the plume is normally positive. Such positive gradient are subtracted from the $\mathrm{XCO}_{2}$ observations, leading to a lower $\mathrm{FFCO}_{2}$ signals (panel b versus a) and a higher $\mathrm{ER}_{\mathrm{CO}}$ (panel e versus d) when correcting for the biogenic impact. The underlying hybrid maps were created using ggmap library in R that adopted the Google Maps (copyright: Map data @2021 Imagery @2021 TerraMetrics). 
Distribution of ERCO [ppb-CO / ppm-CO2] as in In space
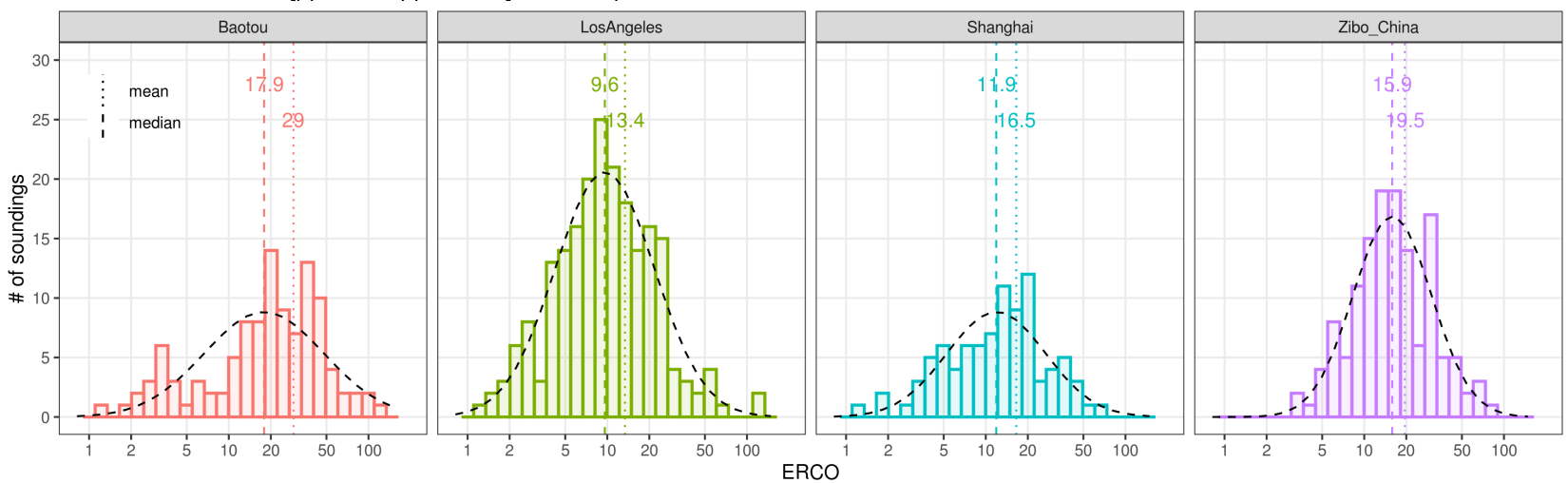

Figure S10. Distribution of ERs across four cities with the x-axis displayed in natural logarithm scale. The log-normal curve is shown as dashed lines. The estimated median/mean values of ERs based on log-normal fit are shown as dashed/dotted vertical lines. Since the ER distribution is stewed towards the lower end, the median values are smaller than the mean values. 

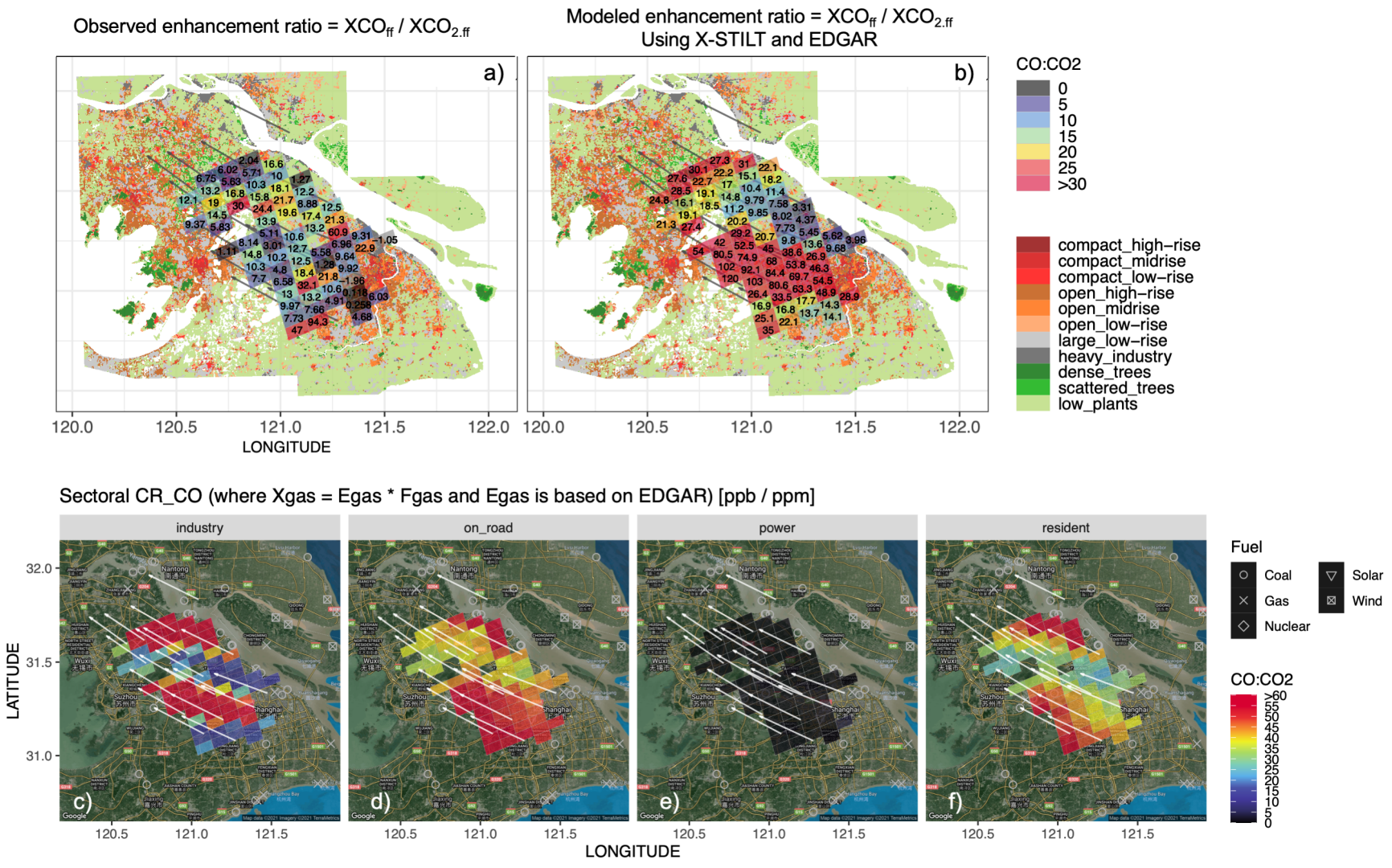

Figure S11. An example of the total FF enhancement ratios [ppb-CO / ppm- $\mathrm{CO}_{2}$ ] observed from OCO-3 and TROPOMI (a) and simulated using X-STILT and EDGARv5 (b) over Shanghai on Feb 20, 2020. Maps of the simulated sectoral enhancement ratios [ppb-CO / ppm- $\mathrm{CO}_{2}$ ] using sectoral emissions from EDGARv5 and X-STILT. Power plant locations from the global power plant dataset are displayed in various symbols with circles indicating the coal-fired power plants. Note that the underlying hybrid maps in the lower panels were created using ggmap library in R that adopted the Google Maps (copyright: Map data (C2021 Imagery (C2021 TerraMetrics). 